\title{
NOTICES
}

\section{Foreign Visiting Professorships of the American Astronomical Society}

Funds provided by the National Science Foundation make it possible for the Society each year to invite four or five distinguished astronomers from overseas to spend an academic semester at an American university or observatory. This program, now in its third year, has been extended for another three years. Its purposes (to enrich U. S. astronomical education and research, and to benefit the visitors in various ways) have been well served by ten Foreign Visiting Professors through July 1962:

W. Becker, Basel, Switzerland, to Indiana, Spring 1960,

P. Bhatnagar, Bangalore, India, to Harvard, Spring 1960,

W. Iwanowska, Torun, Poland, to Perkins Observatory, Spring 1960,

C. Schalen, Lund, Sweden, to Case, Spring 1960,

A. Blaauw, Groningen, Holland, to Vanderbilt, Spring 1961,

M. Fracastoro, Catania, Italy, to Pennsylvania, Fall 1961,

A. Wachmann, Hamburg, Germany, to Columbia, Spring 1961,

A. Beer, Cambridge, England, to Swarthmore, Spring 1962,

A. Hewish, Cambridge, England, to Yale, Spring 1962,

J. Kleczak, Ondrejov, Czechoslovakia, to Colorado, Spring 1962,

Z. Suemoto, Tokyo, Japan, to Michigan, Spring 1962.

A special Committee, appointed by the Council of the Society (now Edmondson, Minkowski, Page, and Strand, with J. M. Chamberlain and Beals, ex officio) considers nominations from any U. S. educational institution engaged in astronomical teaching and research. Invitations are made in the name of the Society by the President; the Society pays travel expenses and a suitable honorarium, and the host institution arranges housing. The Foreign Visiting Professor may teach a course or seminar or supervise student research as well as continuing his own.

Invitations have been accepted by:

W. Fricke, Heidelberg, Germany, for Fall 1962, at Case Institute,

C. W. Allen, London, England, for Spring 1963, at Perkins Observatory,

E. Holmberg, Uppsala, Sweden, for Spring 1963, at Wesleyan,

F. J. Kerr, Sidney, Australia, for Spring 1963, at Texas,

P. Ledoux, Liege, Belgium, for Spring 1963, at Berkeley, California.

Further nominations are invited. Nomination forms may be obtained from the undersigned.

ThORnTON PAGE (Chairman),

Van Vleck Observatory,

Wesleyan University,

Middletown, Connecticut 